\title{
TALEN-mediated Nanog disruption results in less invasiveness, more chemosensitivity and reversal of EMT in Hela cells
}

\author{
Yan Ding ${ }^{1,2}$, Ai Qing $\mathrm{Yu}^{1}$, Cheng Lin $\mathrm{Li}^{1}$, Juan Fang ${ }^{1}$, Yi Zeng ${ }^{2}$ and Dong Sheng $\mathrm{Li}^{1}$ \\ ${ }^{1}$ Hubei Key Laboratory of Embryonic Stem Cell Research, Taihe Hospital, Hubei University of Medicine, Shiyan, Hubei, China \\ ${ }^{2}$ College of Life Science and Bioengineering, Beijing University of Technology, Beijing, China \\ Correspondence to: Dong Sheng Li, email: dsli1698@aliyun.com \\ Yi Zeng, email: zengy@public.bta.net.cn
}

Keywords: Nanog, Hela cell, cervical cancer cell, TALEN, epithelial-mesenchymal transition

Received: June 09, $2014 \quad$ Accepted: July 31, 2014

Published: July 31, 2014

This is an open-access article distributed under the terms of the Creative Commons Attribution License, which permits unrestricted use, distribution, and reproduction in any medium, provided the original author and source are credited.

\section{ABSTRACT}

Emerging evidence suggests that Nanog is involved in cervical tumorigenesis. However, the regulating role of Nanog in tumorigenesis and chemosensitivity are still poorly understood. In this study, Nanog was disrupted by transcription activatorlike effector nucleases (TALEN) in Hela cells and its expression was significantly decreased in a single-cell derived sub-clone with biallelic mutations. The disruption of Nanog not only induced down regulation of some other core transcription factor genes for cell self-renewal, such as Oct4, Sox2 and FoxD3, but also led to the down regulation of some mesenchymal representative genes, vimentin and $\mathbf{N}$-adherin, and up regulation of the epithelial gene, E-cadherin. In addition, the invasiveness and clonogenicity of the Hela cells were obviously affected, and surprisingly their sensitivities to anti-cancer drugs were also significantly increased in vitro. After Xenograft into nude mice, the growth volumes of the neoplasms from the Nanog disrupted Hela cells were significantly smaller compared with those from wild type ones. In conclusion, these results suggest that disruption of Nanog may reverse the status of epithelial-mesenchymal transition, which is critical in tumorigenesis, and alleviate chemoresistance, as well as their invasiveness, in cervical cancer cells.

\section{INTRODUCTION}

The cervical cancers are the third most common cancers in females, with their mortality in the fourth place [1]. Human papilloma virus (HPV) is believed to play the major role in the etiology of these cancers, though some other factors may also be involved [2]. Poor prognosis is usually associated with the characteristics of highly invasive and diffusely metastatic [3]. In the initial stage of invasion and metastasis, the morphogenetic changes due to conversion of polarized epithelial cells to motile mesenchymal cells, are referred as epithelial-mesenchymal transition (EMT). However, how cervical cancer cells acquire the ability to invade surrounding tissues and metastasize is far from understood and little is known about the reversal of EMT in these tumors.

Nanog is a core transcription factor gene for maintaining self-renewal and pluripotency of embryonic stem cells $[4,5]$. It has been reported that abnormal expression of Nanog is associated with human germ cells and several other types of cancers, such as gliomas [6], embryonic carcinomas [7], prostate cancers [8], breast cancers [9], etc. Down regulation of Nanog inhibits the proliferation, colony expansion and clonogenic growth of tumor cells [10], while over expression of Nanog may induce chemoresistance in breast cancer and prostatic cancers [11]. However, the correlation between Nanog expression and the cervical cancer remains unclear and the molecular mechanisms of Nanog in inducing EMT, metastasis and chemoresistance also need to be further clarified.

Since their discoveries, various kinds of RNAi techniques have been widely used to repress some gene expression in vitro because of their convenience in use. However, none of these techniques are able to induce complete disfunction of the target gene. The 
recently developed genome-editing techniques, such as transcription activator-like effector nucleases (TALEN), could overcome this problem [12]. TALEN could induce some mutations at random to a specific gene, which often lead to translational termination and loss of its function [13]. In the present study, Nanog of Hela cells was biallelicly disrupted by TALEN in order to investigate if it plays any role in affecting invasiveness, EMT and chemoresistance in human cervical cancers.

\section{RELSULTS}

\section{T7E1 analysis and selection for cell clones with biallelic mutations}

To assess the endonuclease-dependent genome editing activities, gDNA were prepared from the transfected Hela cells after two rounds of transfection by TALEN. The target region of Nanog gene was amplified by PCR, and then digested by T7E1 enzyme. In result, TALEN induced Nanog mutations were found to be more than $50 \%$ and one cell clone was selected as with biallelic Nanog mutations by gene sequencing from 30 single- cell cultures (Figure 1). As a matter of fact, this biallelic Nanog disrupted Hela cell clone was not true Nanog knockouts due to the polyploidy nature of Hela cells [14, 15]. Obviously, there are at least three Nanog alleles in the selected Hela cell clone based on the gene sequencing results. Nevertheless, they were used for the following experiments.

\section{Nanog disruption alleviated the invasiveness of the Hela cells}

Transwell cell migration assay and scratch assay were carried out to investigate how the invasiveness of Hela cells was affected by Nanog disruption. As shown in Figure 2, the numbers of the cells that passed through the matrigel and transwell at $24 \mathrm{~h}$ and $48 \mathrm{~h}$ were $19 \pm 3$ and $42 \pm 5$ respectively for Nanog disrupted Hela cells, and $58 \pm 7$ and $106 \pm 13$ respectively for those with wild type Nanog. The numbers for Nanog disrupted Hela cells were remarkably less $(\mathrm{P}<0.05)$. In scratch assay, the migration rates for the Nanog disrupted Hela cells at $24 \mathrm{~h}$ and $48 \mathrm{~h}$ were $20 \pm 4 \%$ and $31 \pm 4 \%$ respectively while those for wild type Nanog Hela cells were $29 \pm 4 \%$ and $51 \pm 7 \%$ respectively $(\mathrm{P}<0.05$, Figure 3).
A

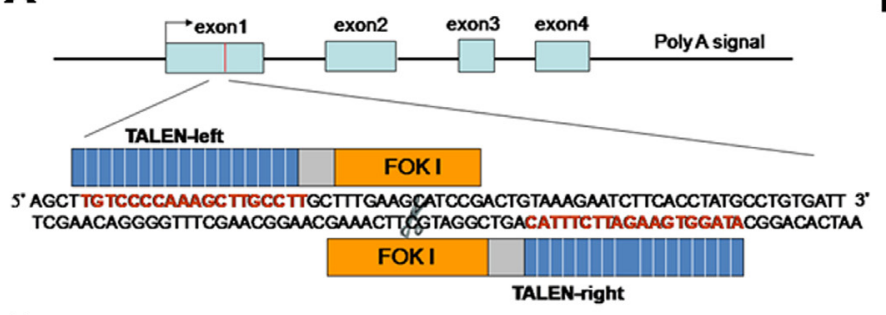

C

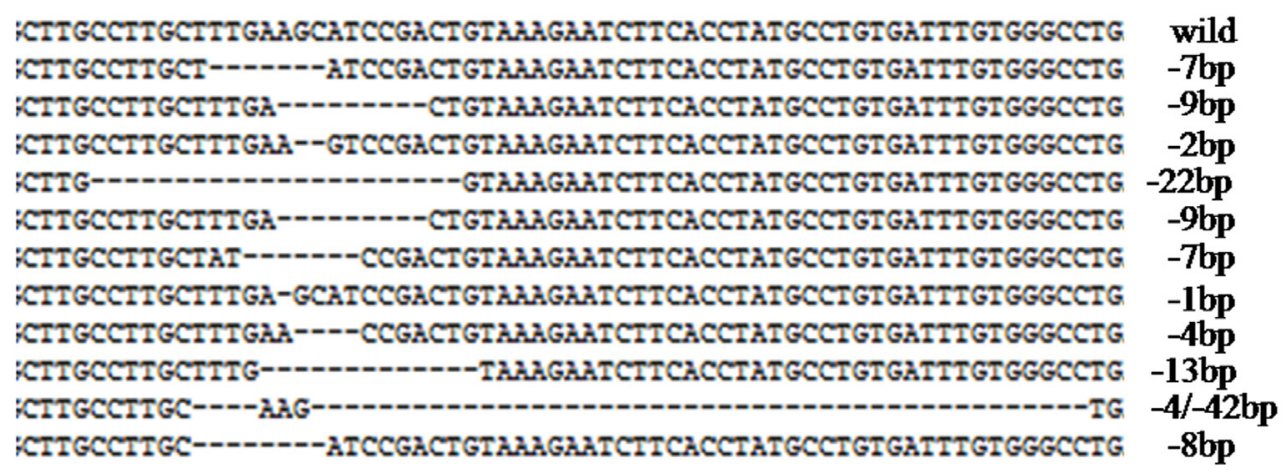

D

Clone 19
$\mathbf{B}$

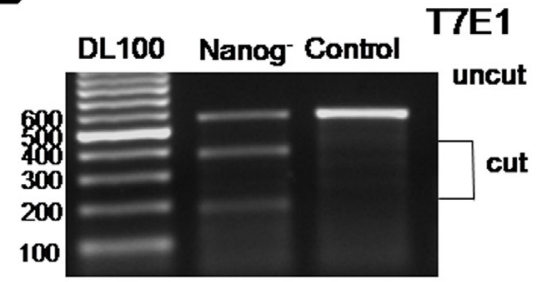

Figure 1: Disruption of Nanog in Hela cells by TALEN. A: Diagrammatic sketch of Nanog and its target site of by TALEN plasmids. B: After two rounds of targeting by TALENs, the target sites of Nanog were amplified by PCR and then digested by T7E1 to evaluate the proportion of the mutated Nanogs. C: Alignment of the sequences of mutated and wild type Nanogs at the TALEN target sites. The number of deleted nucleotides (dashes) was marked on the right end of each sequence. D: Sequencing of Nanog for the selected cell clone after two rounds of targeting by TALEN. This result suggests there be at least three Nanog alleles in Hela cells. Control stands for the Hela cells of wild type; Nanog- stands for those with Nanog disruption. 


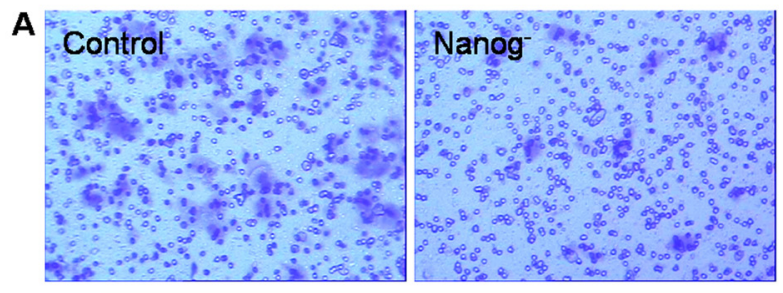

B

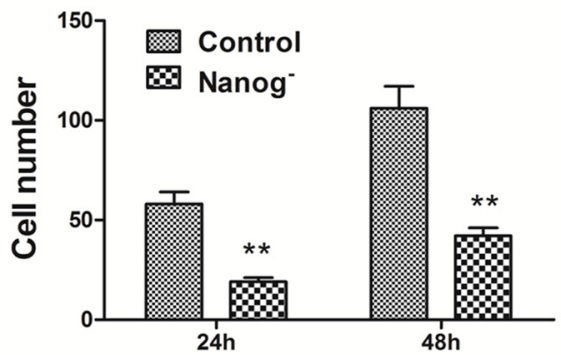

Figure 2: Transwell assay of the Hela cells with/without Nanog disruption. A: the pictures taken by the imaging system. B: Comparison of the cell numbers that passed the membrane of trans-well at 24h and 48h between the Hela cells with/without Nanog disruption. The differences were significant at the both time points. Control stands for the Hela cells of wild type; Nanog- stands for those with Nanog disruption.

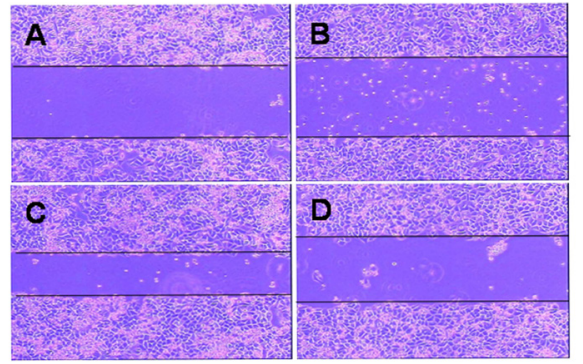

E

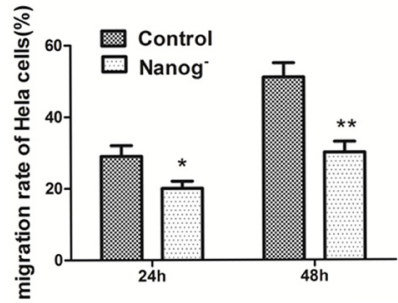

Figure 3: Scratch assay of the Hela cells with/without Nanog disruption. A and C: The pictures taken at $0 \mathrm{~h}$ and 24h for the wild type Hela cells. B and D: The pictures taken at $0 \mathrm{~h}$ and $24 \mathrm{~h}$ for the Hela cells with Nanog disruption. E: Comparison of migration distances between the Hela cells with/ without Nanog disruption at $24 \mathrm{~h}$ and $48 \mathrm{~h}$. The differences were significant both at $24 \mathrm{~h}(* \mathrm{P}<0.05)$ and at $48 \mathrm{~h}(* * \mathrm{P}<0.01)$. Control stands for the Hela cells of wild type; Nanog- stands for those with Nanog disruption.
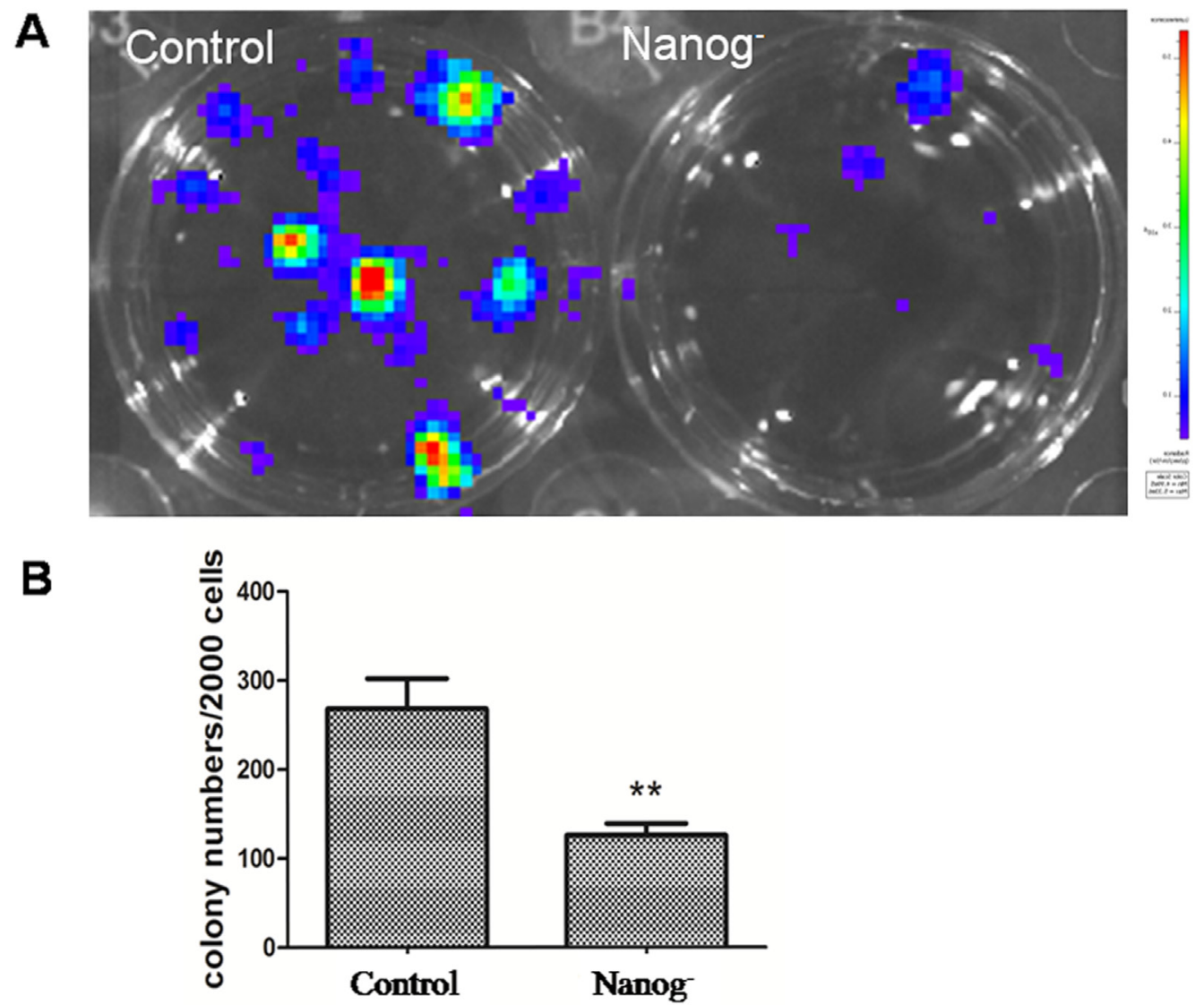

Figure 4: Clonogenicity assay. A: The picture of the cell clones formed from the dissociated Hela cells with/without Nanog disruption after 10 days of incubation at the density of 1000 cells/well in a 12-well plate. B: Comparison of the cell colony numbers formed from dissociated Hela cells with/without Nanog disruption after 12 days of incubation. The colonies formed were significantly fewer for the Hela cells with Nanog disruption (**P<0.01). Control stands for the Hela cells of wild type; Nanog- stands for those with Nanog disruption. 
Nanog disruption decreased the cloning efficiency of the Hela cells

In cell colony formation assay as shown in Figure 4, the colony numbers formed by Nanog disrupted Hela cells were $133 \pm 15$, while those formed by wild type Nanog Hela cells were $285 \pm 32$. This significant difference $(\mathrm{P}<0.05)$ indicated that the clonogenicity of Hela cells was greatly inhibited by Nanog disruption.

\section{Nanog disruption increased the chemosensitivity of the Hela cells}

To evaluate the effect of Nanog disruption on chemosensitivity of Hela cells, both wild type and Nanog disrupted Hela cells were exposed to cisplatin or paclitaxel. In result, Nanog disrupted Hela cells were more sensitive to cisplatin and paclitaxel as shown in Figure $5 \mathrm{~A}, \mathrm{~B}$. The expression of MDR1, which is regarded as an important indicator for drug resistance in chemotherapy, was also significantly down regulated in the Nanog disrupted Hela cells $(\mathrm{P}<0.05)$.

\section{Nanog disruption reversed the status of EMT}

EMT is usually regarded as a progression sign toward malignancy. However, this progress seemed to be reversed by Nanog disruption. Apart from Nanog, several other genes, like Oct4, Sox2 and FoxD3, were also remarkably down regulated in real-time PCR, though the expressions of $c-M y c, \operatorname{Lin} 28$, Klf4 and Rexl remained unchanged (Figure 6A,B). Meanwhile, E-cadherin expression increased while that of vimentin and N-Cadherin decreased in Western blots (Figure 6C). All these changes suggested a reversal of EMT.
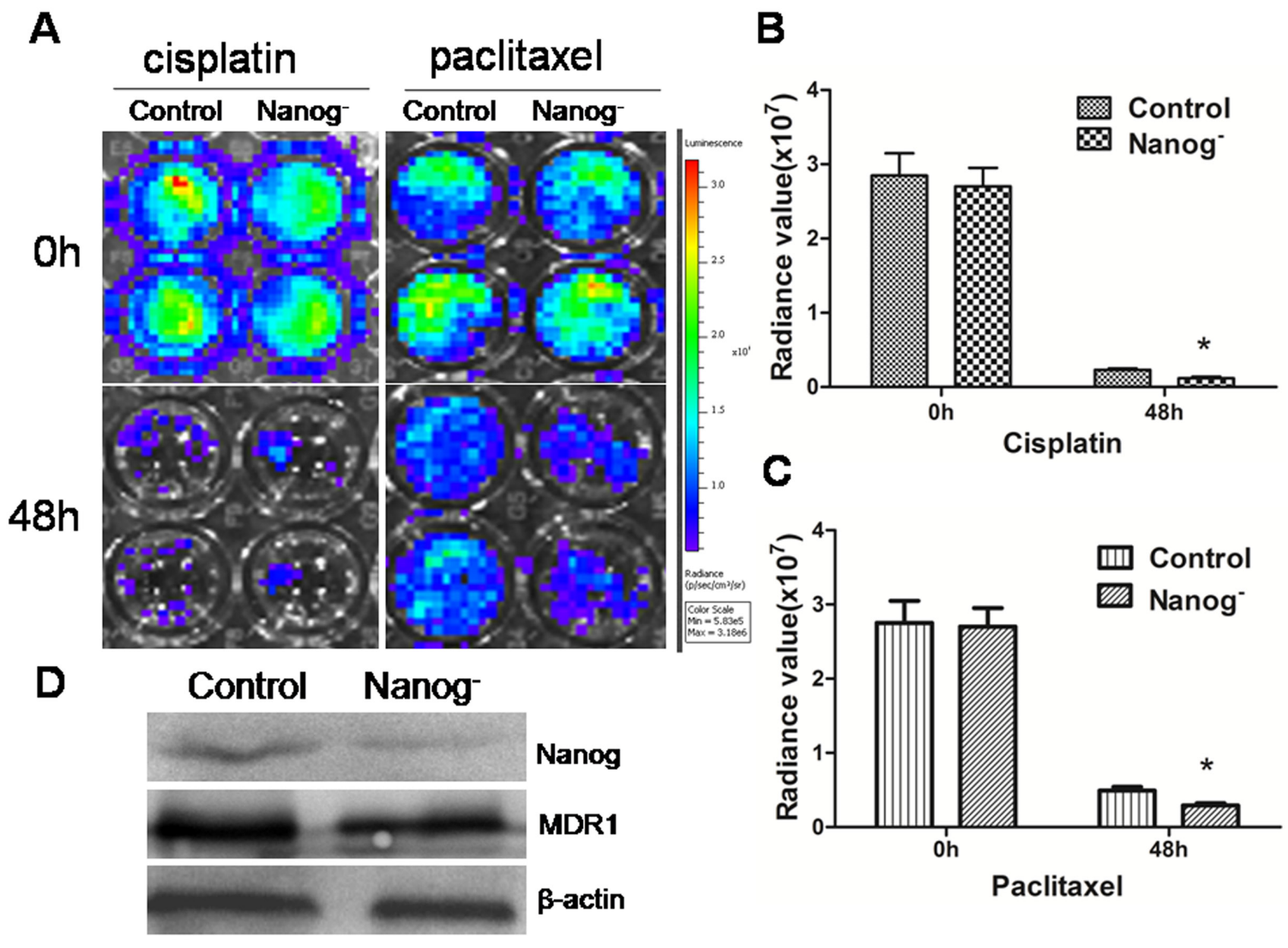

Figure 5: Chemosensitivity assay. The Hela cells with/without Nanog disruption were exposed to cisplatin (1 $\mu \mathrm{g} / \mathrm{ml})$ and paclitaxel $(40 \mathrm{ng} / \mathrm{ml})$ separately for $48 \mathrm{~h}$. The cell viabilities were checked with a florescent imaging system. A: The picture by the imaging system showed that the cell viabilities were decreased more after exposed to both cisplantin and paclitaxel. B and C: Comparisons of the cell viabilities after exposed to cisplatin and paclitaxel respectively. The viabilities of the Hela cells with Nanog disruption were significantly decreased $\left({ }^{*} \mathrm{P}<0.05\right)$. D: Western blot result showed that the level of MDR1 proteins of the Nanog disrupted Hela cells was decreased compared with that of wild type ones. Control stands for the Hela cells of wild type; Nanog- stands for those with Nanog disruption. 

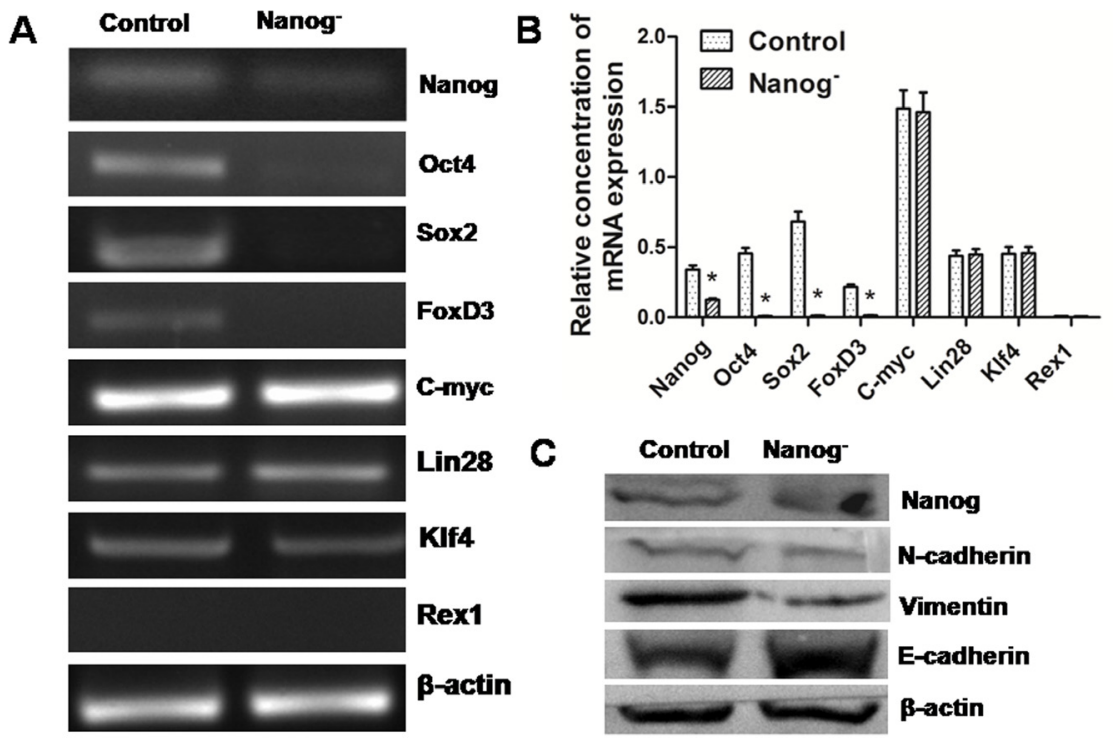

Figure 6: Reversal of EMT in the Nanog disrupted Hela cells. A and B: Real-time PCR results showed that some self-renewal related transcription factor genes such as Oct4, Sox2 and FoxD3 were down regulated by Nanog disruption. C: Western blot results showed that after the disruption of Nanog, the expression of one epithelial marker, E-cadherin, increased, while that of two mesenchymal markers, $\mathrm{N}$-cadherin and Vimentin, was decreased. These results strongly suggest that Nanog disruption could induce the reversal of EMT in Hela cells. Control stands for the Hela cells of wild type; Nanog- stands for those with Nanog disruption.
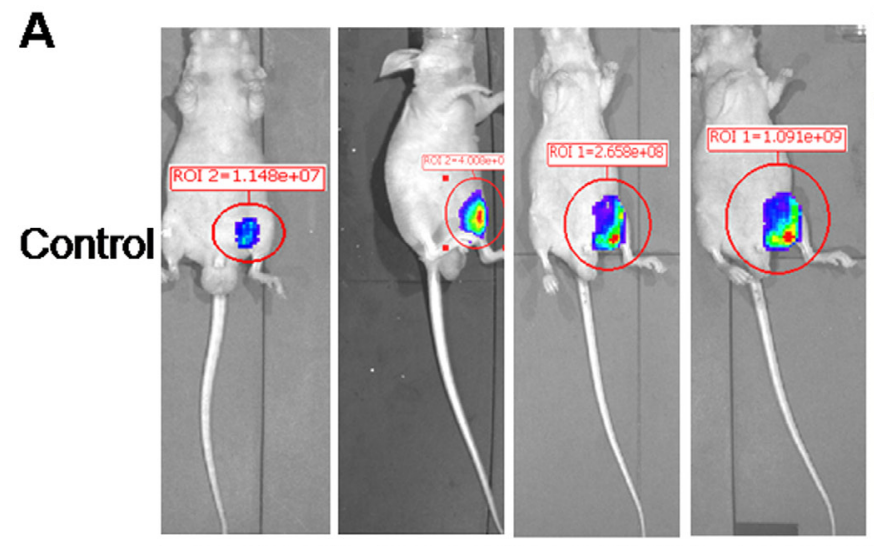

\section{B}
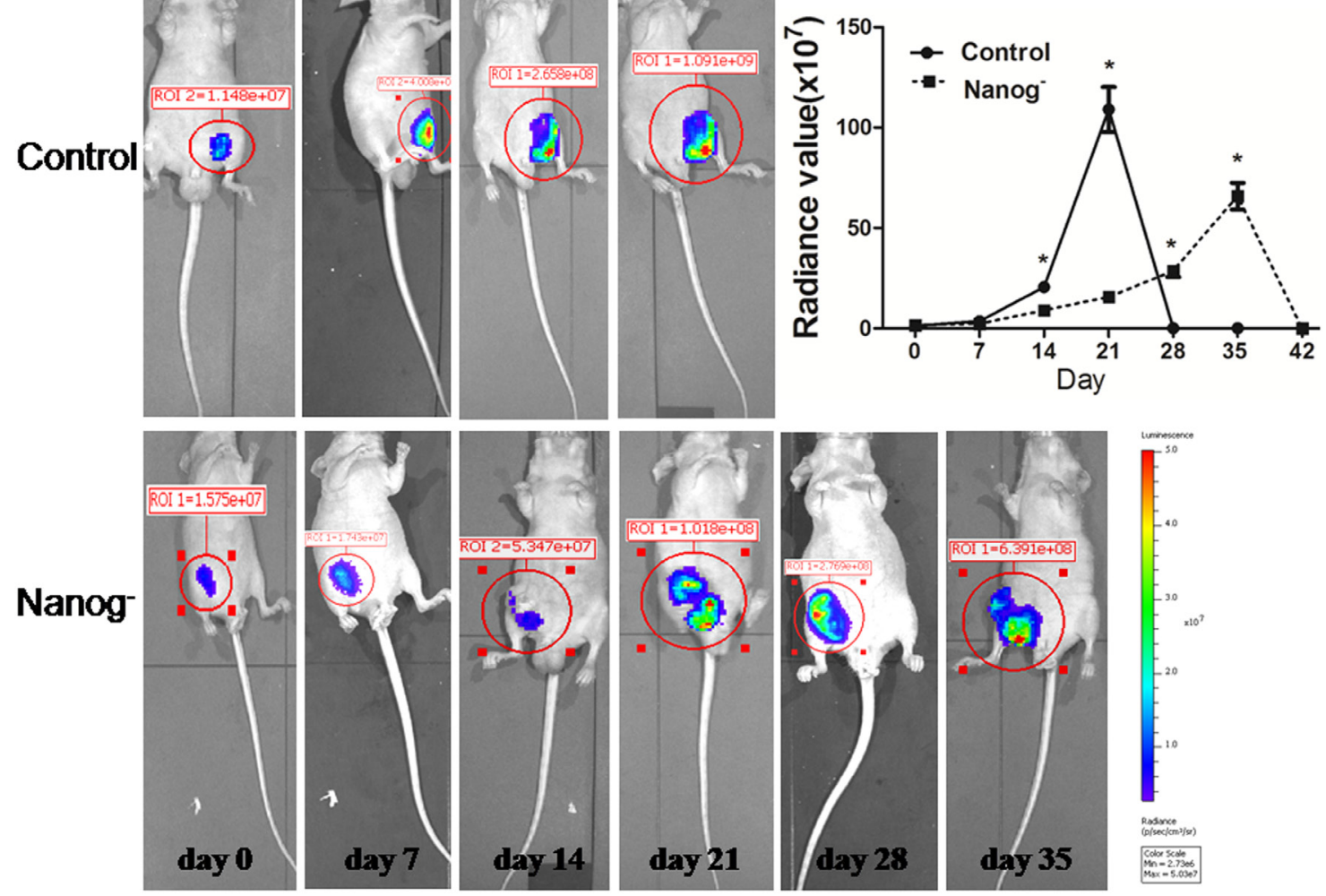

Figure 7: Xenograft test. The Hela cells with/without Nanog disruption (10 million each) were subcutaneously injected into the flanks of nude mice $(n=3)$ and the sizes of neoplasms formed were measured with an in vivo imaging system until mice died (usually $6 \sim 8$ week). A: Serial pictures taken at different time points; B: The changes of fluorescein radiance values of the neoplasms formed from the injected Hela cells with/without Nanog disruption. The difference was significant $(* \mathrm{P}<0.05)$. Control stands for the Hela cells of wild type; Nanogstands for those with Nanog disruption. 


\section{The Nanog disrupted Hela cells grew slower in the Xenograft test}

Both Nanog disrupted and wild type Hela cells were separately injected subcutaneously into nude mice and the sizes of neoplasms formed from implanted Hela cells were measured weekly. In result, the size of the neoplasms formed from Nanog disrupted Hela cells was significantly smaller than those from the wild type ones $(\mathrm{P}<0.05)$. Furthermore, the mice implanted with Nanog disrupted Hela cells lived longer than those with wild type ones (6-8 weeks vs. 4-5 weeks) (Figure 7).

\section{DISCUSSION}

The development of a malignant tumor is a complex process of multiple factors and stages, involving malfunctions or mutations of a variety of genes. The EMT is believed to be one of the most critical steps for neoplasms to progress toward malignancy. It endows tumor cells with migratory and invasive properties [16]. Understanding the molecular mechanism underlines EMT and finding a way to stop or reverse it should be very important for developing new therapeutic strategies against various cancers.

In this study, Nanog was disrupted by TALEN and that seemed to result in a dramatic reversal of EMT process in Hela cells. Not only the expression of Nanog was remarkably inhibited, but also the expressions of some other core transcription factor genes for cell self-renewal, such as Oct4, Sox2 and FoxD3, were down regulated. E-cadherin mediates epithelial cell-to-cell adhesion [17]. The decrease of E-cadherin expression is a critical step in the process of EMT [18], and it is often companied with increase of $\mathrm{N}$-cadherin expression. Vimentin is one of the major mesenchymal intermediate filaments and its expression represents the completely dedifferentiated state in tumor cells that are highly proliferative and invasive [19]. Remarkably, the E-cadherin expression increased while the expressions of $\mathrm{N}$-cadherin and vimentin decreased after disruption of Nanog. These data suggest Nanog may play a critical role in the process of EMT.

The grade of malignancy is closely related with the invasiveness and the clonogenicity of a tumor. Both transwell cell migration assay and scratch assay showed that the invasiveness of the Nanog disrupted Hela cells was significantly alleviated. Also, the clonogenicity of Hela cells was obviously affected in the cell colony formation assay. These findings suggested that the Nanog disrupted Hela cells become less malignant, which is very well in line with the phenomenon described above as a reversal of EMT process.

Chemotherapy has been widely used in the treatment of a variety of cancers including cervical cancers. However, the benefits are frequently limited by the chemoresistance of cancer cells through different signaling pathways, such as PI3K/AKT and MAPK (mitogen-activated protein kinase) [20, 21]. Studies have shown that the failure of chemotherapy in many malignant tumors was partially associated with abnormal expression of MDR1 gene, which encodes the P-glycoprotein to pump anticancer agents out of the cells $[22,23]$. Surprisingly in this study, MDR1, the most important indicator for chemoresistance in chemotherapy, was down regulated by Nanog disruption, though the mechanism underlined remained unclear. And these Hela cells did show more chemosensitivity when exposed to either cisplatin or paclitaxel.

In conclusion, our data suggested that the disruption of Nanog could reverse the status of EMT, which resulted in alleviation of invasiveness and poor clonogenicity in Hela cells, and that MDR1, an indicator gene for chemoresistance, could somehow be down regulated by even partial Nanog disruption, though it is usually thought to be regulated through other singling pathways, and this down regulation of MDR1 did result in less chemoresistance.

\section{MATERIALS AND METHODS}

\section{Cell Culture}

The Hela cells transfected with a firefly luciferase gene (also named as Hela-luc cells) was a generous gift from Dr. Wang (University of British Columbia), were maintained in Dulbecco's modified Eagle's medium (DMEM) containing 10\% fetal bovine serum (FBS).

\section{TALEN plasmids construction and cell transfection}

The paired Nanog TALEN arms were designed according to the manufacture's instruction (Figure 1A). TALEN plasmids were constructed by one-step ligation using the Fast TALE ${ }^{\mathrm{TM}}$ TALEN Assembly Kit (SIDANSAI).

About $10 \mu \mathrm{g}$ of paired Nanog TALEN plasmids (each $5 \mathrm{ug}$ ) were mixed with $1 \times 10^{6}$ Hela cells in the cuvette with $100 \mu$ l Opti-MEM. The transfection was carried out under $130 \mathrm{~V}$ by NEPA21 (Japan). The transfected cells were transferred into one well of 6-well plate and cultured at $37^{\circ} \mathrm{C}$ against puromycin at $3 \mu \mathrm{g} / \mathrm{ml}$. After 3 days, the cells were refreshed with the medium free of puromycin and kept at $37^{\circ} \mathrm{C}$ for few more days before harvested for DNA extraction and single-cell culture in order to select the Nanog disrupted cell clones. 
Table 1: Primers used for RT-PCR.

\begin{tabular}{|l|l|c|}
\hline Gene & Sequence (forward; reverse) & Product length (bp) \\
\hline$\beta$-actin & F: CTGGAACGGTGAAGGTGACA & 140 \\
\hline Nanog & R: AAGGGACTTCCTGTAACAACGCA & 104 \\
\hline Oct4 & R: TTGCTCCTCTTCCTTCCTCCAT & 193 \\
\hline & F: GACAACAATGAGAACCTTCAGGAGA & 215 \\
\hline Sox2 & R: TTCTGGCGCCGGTTACAGAACCA & \\
\hline & F: CCCCCGGCGGCAATAGCA & 306 \\
\hline Rexl & R: TCGGCGCCGGGGAGATACAT & 180 \\
\hline & F: CAGATCCTAAACAGCTCGCAGAAT & \\
\hline c-Myc & R: GCGTACGCAAATTAAAGTCCAGA & 392 \\
\hline & F: GATTCTCTGCTCTCCTCGAC & \\
\hline Klf4 & R: TCCAGACTCTGACCTTTTGC & 153 \\
\hline & F: ACGATCGTGGCCCCGGAAAAGGA & \\
\hline Lin28 & R: GTAGTGCTTTCTGGCTGGGCTCC & 161 \\
\hline & F: CCAGGCAAAAAGATCTGAAA & \\
\hline FoxD3 & R: AGAAAAGAGGGCAGGGTAGA & \\
\hline & F: GACGACGGGCTGGAAGAGAA & \\
\hline & R: GCCTCCTTGGGCAATGTCA & \\
\hline
\end{tabular}

\section{T7 endonuclease 1 (T7E1) assay}

Genomic DNA was prepared from both the Nanog disrupted and wild type Hela cells with Blood Genomic DNA Extraction Mini Kit (TIANGEN). The genomic region encompassing the TALEN target site was amplified by PCR and its products were denatured and annealed to form heteroduplex DNA. The annealed DNA was treated with 5 units of T7E1 (Viewsolid Biotech) at $37^{\circ} \mathrm{C}$ for $15 \mathrm{~min}$ and then ran an agarose gel to separate the DNA fragments. The TALEN target rate was calculated on the intensities of DNA bands, which were proportionally measured by grey scale technique.

\section{Scratch assay}

The scratch assay [2] was used to observe the cell migration. The Hela cells with/without Nanog disruption were cultured to completely confluent in 6-well plates and a scratch was made across the cell monolayer of each well with a $10 \mu 1$ pipette tip. Then washed the cell monolayer 3 times with D-PBS and incubated in serum free DMEM at $37{ }^{\circ} \mathrm{C}$ with $5 \% \mathrm{CO}_{2}$ for $48 \mathrm{~h}$. The widths of the scratch were measured and the percentages of narrow down were compared at $0 \mathrm{~h}, 24 \mathrm{~h}$ and $48 \mathrm{~h}$ respectively. The experiment was performed in triplicates.

\section{Cell migration assay}

Cell migration assays were carried out with transwell filters as described by Liu et al [2]. Both the
Hela cells with Nanog disruption and those without were digested and re-suspended with DMEM medium to $1 \times 10^{5}$ cells $/ \mathrm{ml}$. $200 \mu \mathrm{l}$ cell suspension were seeded into an upper chamber of the polycarbonate membrane filter inserts with $8-\mu \mathrm{m}$ pores (Corning, USA), which were pre-coated with matrigel. The lower chamber was filled with $500 \mu$ of DMEM medium with $10 \%$ FBS. The cells (not migrated) in the upper chamber surface were removed with cotton swabs after incubated at $37^{\circ} \mathrm{C}$ for 24 and $48 \mathrm{~h}$, and the cells (migrated) on the bottom side of the membrane were fixed with $95 \%$ ethanol for $30 \mathrm{~min}$, stained with the $0.1 \%$ crystal violet and counted under a microscope. The experiment was performed in triplicates.

\section{Chemosensitivity assay}

The chemosensitivities of the Hela cells with/ without Nanog disruption were compared by the cell viabilities after exposed to cisplatin or paclitaxel. Briefly, after washed with PBS, freshly disassociated cells were seeded into a 96 -well plate $\left(1 \times 10^{4}\right.$ cells/well $)$ and incubated at $37^{\circ} \mathrm{C}$ for $24 \mathrm{~h}$. Refreshed the cells with the growth media containing $1 \mu \mathrm{g} / \mathrm{ml}$ cisplatin or $40 \mathrm{ng} / \mathrm{ml}$ cisplatin and continually incubated for 48 more hours, took the cells out of the incubator, added 1ul luciferase substrate to the medium and left them at RM for10-15 $\mathrm{min}$, then checked cell viabilities with a florescent imaging system (IVIS ${ }^{\circledR}$ Spectrum, caliper). The experiment was performed in duplicates and repeated three times. 


\section{Clonogenicity assay}

The clonogenicity was analyzed by colonyformation tests. The Hela cells with/without Nanog disruption were plated into 6 wells at 1000 cells/well in a 12 -well plate pre-coated with $1 \%$ gelatin. The cells were incubated at $37^{\circ} \mathrm{C}$ for 10 to 12 days, until the cell colonies grew large enough to be visualized. Then the colonies were counted with the same imaging system as the chemosensivity assay used. The experiment was performed in triplicates.

\section{Xenograft test}

The Hela cells with/without Nanog disruption were subcutaneously injected into nude mice (3 mice each group) separately. These mice were bred and the sizes of the neoplasms from implanted cells were checked weekly until the mice died with the same as the chemosensivity assay used.

\section{Quantitative PCR}

The cells were harvested with TRIzol (Invitrogen) and total RNA was isolated according to the manufacturer's instructions. The cDNA were synthesized by using a reverse transcription kit (Takara Bio, China), and the quantitative PCR was conducted with a SYBR green one (Takara Bio, China). The PCR reaction proceeded as follows: $95^{\circ} \mathrm{C}$ for $30 \mathrm{sec}$, then 40 cycles including $95^{\circ} \mathrm{C}$ for $30 \mathrm{sec}, 60^{\circ} \mathrm{C}$ for $30 \mathrm{sec}$ and $72^{\circ} \mathrm{C}$ for $30 \mathrm{sec}$. The fold changes in gene expressions were normalized to $\beta$-actin. Gene-specific primers sets are shown in the Table 1.

\section{Western blot}

Cells of each group were collected. The proteins were extracted and separated by SDS-PAGE, and then transferred onto PVDF membranes. After been blocked with $1 \% \mathrm{BSA}$ for $1 \mathrm{~h}$ at room temperature (RT), the membranes were then incubated with antibodies against Nanog (1:200, Abcam), Vimentin(1:200, Santa Cruze) E-cadherin (1:200, Santa Cruze), N-cadherin (1:200, Santa Cruze), MDR1 (1:200, Santa Cruze), and $\beta$-actin (1:1000, TIANGEN) at $4^{\circ} \mathrm{C}$ overnight. After washing 3 times with TBST, the membranes were incubated with alkaline phosphatase-conjugated secondary antibody (1:500, TIANGEN) at RT for $1 \mathrm{~h}$. Then washed the membranes 3 times with TBST and imaged with a gel imaging system (BIO-RAD).

\section{STATISTICAL ANALYSIS}

Results were presented as means of three independent experiments $(\mathrm{M} \pm \mathrm{SD})$. Statistical analyses were performed with the Student's t-test by using SPSS 13.0. $\mathrm{P}<0.05$ was considered statistically significant.

\section{ACKNOWLEDGEMENTS}

This study was supported by the grants from Ministry of Science and Technology of China (2013ZX10001004-002-005), Science and Technology Department of Hubei Province (2012FFA37) and Science and Technology Department of Shiyan City (069S, 2013)

\section{CONFLICTS OF INTEREST}

The authors declare no conflict of interest.

\section{REFERENCES}

1. Jemal A1, Bray F, Center MM, Ferlay J, Ward E, Forman D. Global cancer statistics. CA Cancer J Clin. 2011; 61: 6990.

2. Liu X, Wang D, Liu H, Feng Y, Zhu T, Zhang L, Zhu B, Zhang Y. Knockdown of astrocyte elevated gene-1 (AEG1) in cervical cancer cells decreases their invasiveness, epithelial to mesenchymal transition, and chemoresistance. Cell Cycle. 2014; 13:11, 1-6.

3. Kim CJ, Jeong JK, Park M, Park TS, Park TC, Namkoong SE, Park JS. HPV oligonucleotide microarray-based detection of HPV genotypes in cervical neoplastic lesions. Gynecol Oncol. 2003; 89:210-217.

4. Chambers I, Colby D, Robertson M, Nichols J, Lee S, Tweedie S, Smith A. Functional expression cloning of Nanog, a pluripotency sustaining factor in embryonic stem cells. Cell. 2003; 113: 643-655.

5. Mitsui K, Tokuzawa Y, Itoh H, Segawa K, Murakami M, Takahashi K, Maruyama M, Maeda M, Yamanaka S. The homeoprotein Nanog is required for maintenance of pluripotency in mouse epiblast and ES cells. Cell. 2003; 113: 631-642.

6. Zbinden M, Duquet A, Lorente-Trigos A, Ngwabyt SN, Borges I, Ruiz i Altaba A. NANOG regulates glioma stem cells and is essential in vivo acting in a cross-functional network with GLI1 and p53. EMBO J. 2010; 29: 26592674.

7. Freberg CT, Dahl JA, Timoskainen S, Collas P. Epigenetic reprogramming of OCT4 and NANOG regulatory regions by embryonal carcinoma cell extract. Mol Biol Cell. 2007; 18: 1543-1553.

8. Gu G, Yuan J, Wills M, Kasper S. Prostate cancer cells with stem cell characteristics reconstitute the original human 
tumor in vivo. Cancer Res. 2007; 67: 4807-4815.

9. Ben-Porath I, Thomson MW, Carey VJ, Ge R, Bell GW, Regev A, Weinberg RA. An embryonic stem cell-like gene expression signature in poorly differentiated aggressive human tumors. Nat Genet. 2008; 40: 499-507.

10. Jeter CR, Badeaux M, Choy G, Chandra D, Patrawala L, Liu C, Calhoun-Davis T, Zaehres H, Daley GQ, Tang DG. Functional evidence that the self-renewal gene NANOG regulates human tumor development. Stem Cells. 2009; 27: 993-1005.

11. Jeter CR, Liu B, Liu X, Chen X, Liu C, Calhoun-Davis T, Repass J, Zaehres H, Shen JJ, Tang DG. NANOG promotes cancer stem cell characteristics and prostate cancer resistance to androgen deprivation. Oncogene. 2011; 30: 3833-3845.

12. Miller JC, Tan S, Qiao G, Barlow KA, Wang J, Xia DF, Meng X, Paschon DE, Leung E, Hinkley SJ, Dulay GP, Hua KL, Ankoudinova I, et al. A TALE nuclease architecture for efficient genome editing. Nat Biotechnol. 2011; 29: 143148.

13. Cade L, Reyon D, Hwang WY, Tsai SQ, Patel S, Khayter C, Joung JK, Sander JD, Peterson RT, Yeh JR. Highly efficient generation of heritable zebrafish gene mutations using homo- and heterodimeric TALENs. Nucleic Acids Res. 2012; 40: 8001-8010.

14. Landry JJ, Pyl PT, Rausch T, Zichner T, Tekkedil MM, Stütz AM, Jauch A, Aiyar RS, Pau G, Delhomme N, Gagneur J, Korbel JO, Huber W, Steinmetz LM. The Genomic and Transcriptomic Landscape of a HeLa Cell Line. G3 (Bethesda). 2013; 3: 1213-1224.

15. Jeter CR, Badeaux M, Choy G, Chandra D, Patrawala L, Liu C, Calhoun-Davis T, Zaehres H, Daley GQ, Tang DG. The haplotype-resolved genome and epigenome of the aneuploid HeLa cancer cell line. Nature. 2013; 500: 207211.

16. Mani SA, Guo W, Liao MJ, Eaton EN, Ayyanan A, Zhou AY, Brooks M, Reinhard F, Zhang CC, Shipitsin M, Campbell LL, Polyak K, Brisken C, et al. The epithelialmesenchymal transition generates cells with properties of stem cells. Cell. 2008; 133:704-715.

17. Gumbiner BM. Regulation of cadherin adhesive activity. $\mathrm{J}$ Cell Biol. 2000; 148:399-404.

18. Hao L, Ha JR, Kuzel P, Garcia E, Persad S. Cadherin switch from $\mathrm{E}$ - to $\mathrm{N}$-cadherin in melanoma progression is regulated by the PI3K/PTEN pathway through Twist and Snail. Br J Dermatol. 2012; 166:1184-1197.

19. Hendrix MJ, Seftor EA, Chu YW, Trevor KT, Seftor RE. Role of intermediate filaments in migration, invasion and metastasis. Cancer Metastasis Rev. 1996; 15:507-525.

20. Sagawa Y, Fujitoh A, Nishi H, Ito H, Yudate T, Isaka $\mathrm{K}$. Establishment of three cisplatin-resistant endometrial cancer cell lines using two methods of cisplatin exposure. Tumour Biol. 2011; 32:399-408.

21. Ding ZY, Liu GH, Olsson B, Sun XF. Upregulation of the antiapoptotic factor Livin contributes to cisplatin resistance in colon cancer cells. Tumour Biol. 2013; 34:683-693.

22. Pérez-Tomás R. Multidrug resistance: retrospect and prospects in anti-cancer drug treatment. Curr Med Chem. 2006; 13: 1859-1876.

23. Goda K, Bacsó Z and Szabó G. Multidrug resistance through the spectacle of P-glycoprotein. Curr Cancer Drug Targets. 2009; 9: 281-297. 